\title{
Desafios da família cuidando da pessoa com transtorno mental
}

RESUMO | Objetivo: Verificar na literatura científica os desafios enfrentados por familiares no cuidado da pessoa com Transtorno Mental. Método: Trata-se de uma revisão integrativa. Realizou-se a busca por artigos; com delimitação nos últimos 5 anos (20152020); nos idiomas português, inglês e espanhol; disponíveis na íntegra. Nas seguintes plataformas de dados: DOAJ, LILACS, MEDLINE, SciELO, SCOPUS e Web of Science. Resultados: Os dados foram organizados e apresentados em figuras e tabelas. Dos 5740 estudos encontrados, 160 estava disponível na DOAJ, 123 na LILACS, 3056 na MEDLINE, 28 na SciELO, 834 na SCOPUS e 1539 na Web of Science. Contudo, após a leitura permaneceram apenas os que atendiam aos critérios para inclusão e exclusão descritos na metodologia, 9 estudos. Conclusão: Observa-se que as instituições não estão prontas para colocar pessoas com transtorno mental na rua, devolver para suas residências ou expor para residências terapêuticas, sem nenhum apoio da rede. Descritores: Transtornos Mentais; Família; Cuidadores.

ABSTRACT | Objective: To check the scientific literature the challenges faced by family members in the care of people with Mental Disorders. Method: This is an integrative review. The search for articles was carried out; with delimitation in the last 5 years (20152020); in Portuguese, English and Spanish; available in full. On the following data platforms: DOAJ, LILACS, MEDLINE, SciELO, SCOPUS and Web of Science. Results: Data were organized and presented in figures and tables. Of the 5740 studies found, 160 were available from DOAJ, 123 from LILACS, 3056 from MEDLINE, 28 from SciELO, 834 from SCOPUS and 1539 from the Web of Science. However, after reading, only those who met the criteria for inclusion and exclusion described in the methodology remained, 9 studies. Conclusion: It is observed that institutions are not ready to put people with mental disorders on the street, return them to their homes or expose them to therapeutic homes, without any support from the network.

Descriptors: Mental Disorders; Family; Caregivers.

RESUMEN | Objetivo: Verificar la literatura científica sobre los desafíos que enfrentan los familiares en el cuidado de personas con Trastornos Mentales. Método: Esta es una revisión integradora. Se realizó la búsqueda de artículos; con delimitación en los últimos 5 años (2015-2020); en portugués, inglés y español; disponible en su totalidad. En las siguientes plataformas de datos: DOAJ, LILACS, MEDLINE, SCIELO, SCOPUS y Web of Science. Resultados: Los datos se organizaron y presentaron en figuras y tablas. De los 5740 estudios encontrados, 160 estaban disponibles en DOAJ, 123 en LILACS, 3056 en MEDLINE, 28 en SciELO, 834 en SCOPUS y 1539 en Web of Science. Sin embargo, después de la lectura, solo quedaron los que cumplieron con los criterios de inclusión y exclusión descritos en la metodología, 9 estudios. Conclusión: Se observa que las instituciones no están preparadas para poner a las personas con trastornos mentales en la calle, devolverlas a sus hogares o exponerlas a hogares terapéuticos, sin ningún apoyo de la red.

Descriptores: Transtornos Mentales; Família; Cuidadore.

\section{Maria Luiza Figueroa de Albuquerque Aymar}

Especialista em Enfermagem em Saúde Mental pelo Instituto de Desenvolvimento Educacional (IDE). Especialista em Enfermagem Obstétrica pela Fundação de Ensino Superior de Olinda (FUNESO). Recife, Pernambuco (PE), Brasil.

ORCID : 0000-0003-2427-2687

\section{Marta Maria Francisco}

Mestra em Enfermagem pela Universidade Federal de Pernambuco (UFPE). Recife, Pernambuco (PE), Brasil.

ORCID: 0000-0001-8938-9179

\section{Iara Alves Feitoza de Andrade}

Mestra em Enfermagem pela Universidade Federal de Pernambuco (UFPE). Especialista em Enfermagem em Hematologia pela Universidade de Pernambuco (UPE). Reci- fe, Pernambuco (PE), Brasil. ORCID: :0000-0003-3495-0613

\section{Liniker Scolfild Rodrigues da Silva}

Especialista em Saúde Mental, Álcool e outras Drogas pela Faculdade ALPHA. Sanitarista, Especialista em Saúde Coletiva na modalidade Residência pela Faculdade de Ciências Médicas (FCM)/UPE. Recife, Pernambuco (PE), Brasil.

ORCID: 0000-0003-3710-851X

\section{Renata Rosal Lopes da Cruz}

Especialista em Análise de Situação de Saúde pela Universidade Federal de Goiás (UFG). Sanitarista pelo Instituto Brasileiro de Pós-Graduação e Extensão (IBPEX). Recife, Pernambuco (PE), Brasil. ORCID ID: 0000-0001-7222-6875

\section{Elânia Maria da Silva Simões \\ Especialista em Enfermagem em Infectolo- gia na modalidade Residência pela Facul- dade de Enfermagem Nossa Senhora das Graças/Universidade de Pernambuco (FEN- GS/UPE). Recife, Pernambuco (PE), Brasil. ORCID ID: :0000-0003-3328-1737}

Recebido em: 01/09/2021

Aprovado em: 01/12/2021 
INTRODUÇÃO

$\longrightarrow$ modelo manicomial encontrava-se em ressonância com as Políticas de Saúde do Brasil que tiveram seu marco na $8^{\text {a }}$ Conferência Nacional de Saúde (1986), na $1^{\text {a }}$ Conferência Nacional de Saúde Mental (1987), na $2^{a}$ Conferência Nacional de Saúde Mental (1992), culminando para $3^{\text {a }}$ Conferência Nacional de Saúde Mental (2001) (1).

A família acometida com o parente com transtorno mental implica considerar a transformação da pessoa outrora conhecida com comportamentos de isolamento, autodestruição, agressividade e hábitos inadequados de higiene, que podem gerar ao cuidador ansiedade, raiva, culpa, medo e angústia. Além das atribuições adquiridas como acompanhá-los regularmente aos serviços de saúde, administrar a medicação, fornecer suporte social, arcar com gastos dos tratamentos e superar as dificuldades dessas tarefas, estimular o convívio social, passar por constrangimentos, preconceitos e estigmas em relação à pessoa acometida. Assim, o cuidador predispõe-se ao desenvolvimento de sofrimento mental e comprometimento da vida social, ocupacional e financeira, pois as demandas da pessoa com transtorno mental podem tornar-se uma experiência vivida como sobrecarga para o cuidador (2).

A Política Nacional de Saúde Mental propõe que as práticas de saúde mental na atenção básica substituam o modelo tradicional medicalizante. Portanto, é necessária a articulação da rede de cuidados visando à integralidade desse indivíduo na atenção básica e na estratégia de saúde da família, sendo essas a porta de entrada preferencial de todo o Sistema de Saúde, inclusive no que diz respeito às necessidades de saúde mental desses usuários (3). Porém, cabe ainda à família o papel do cuidador que é o indivíduo que se dispõe em favor de cuidados necessários ao enfermo, muitas vezes expondo-se a

\section{A família acometida} com o parente com transtorno mental implica considerar a transformação da pessoa outrora conhecida com comportamentos de isolamento, autodestruição, agressividade e hábitos inadequados de higiene, que podem gerar ao cuidador ansiedade, raiva, culpa, medo e angústia riscos de comprometimento de sua própria saúde em benefício do doente (4).

Essa pesquisa surgiu da necessidade de um familiar que cuida da pessoa vivendo com transtorno mental descrever as dificuldades enfrentadas ao cuidar do ente querido e sua angústia na ineficácia, muitas vezes, do atendimento junto aos serviços de saúde, de educação e da rede social de apoio. O que se desvia dos princípios do Sistema Único de Saúde (SUS) e acordos firmados nos marcos históricos, citados anteriormente. A saúde mental desse familiar é fundamental, principalmente no que concerne a sua estrutura, pois em alguns momentos poderá se apresentar desestruturada diante dos desafios surgidos diariamente. Portanto, não só o paciente como também o familiar que convive deverá receber um suporte da rede de apoio para que essa família seja assistida.

Nesse contexto, o objetivo principal desta pesquisa foi verificar na literatura científica os desafios enfrentados por familiares no cuidado da pessoa com Transtorno Mental.

MÉTODO

Este se trata de um estudo bibliográfico, descritivo do tipo revisão integrativa, com abordagem qualitativa que oferece oportunidades para verificar a literatura científica e entender os amplos tópicos de pesquisa, contribuindo para as práticas de assistência ao paciente com base no conhecimento científico (5).

A partir das seguintes etapas: (1) elaboração da questão norteadora e objetivo do estudo; (2) definição de critérios de inclusão e exclusão das produções científicas; (3) busca de estudos científicos nas bases de dados e bibliotecas virtuais; (4) análise e categorização das produções encontradas; (5) resultados e discussão dos achados (6).

Para o levantamento da questão norteadora, utilizou-se a estratégia 
PICo, uma metodologia que auxilia na construção de uma pergunta de pesquisa e busca de evidências para uma pesquisa não-clínica, onde $\mathrm{P}=$ População/Paciente; I = Interesse; e Co = Contexto (P: Cuidador familiar; I: Desafios enfrentados pela família; Co: Saúde do cuidador que presta assistência a pessoa com transtorno mental). Desta forma, definiu-se a seguinte questão norteadora da pesquisa: "Quais os desafios enfrentados pela família cuidando da pessoa com transtorno mental?".

Para seleção dos artigos, utilizaram-se como critérios de inclusão: artigo original, disponível na íntegra, publicado nos últimos 5 anos (2015-2020) em português, inglês ou espanhol, que respondessem ao objetivo do estudo. Foram excluídas as literaturas cinzas, bem como publicações repetidas de estudos em mais de uma base de dados e os artigos que não responderam à questão norteadora do estudo e que possibilitasse o acesso pelo Virtual Private Network (VPN) da Universidade de Pernambuco (UPE). Justifica-se a delimitação temporal nos últimos 5 anos visando o levantamento de artigos mais recentes.

O levantamento dos dados ocorreu durante o mês de junho e julho de 2021 nas seguintes Bases de Dados: Directory of Open Access Journals (DOAJ); Literatura Latino-Americana e do Caribe em Ciências da Saúde (LILACS); Medical Literature Analysis and Retrieval System Online (MEDLINE); SCOPUS, e Web of Science, bem como na Biblioteca Virtual Scientific Electronic Library Online (SciELO).

Buscaram-se os artigos indexados a partir dos Descritores em Ciências da Saúde (DeCS): "Transtornos Mentais", "Família", "Cuidadores". Utilizaram-se os respectivos termos provenientes do Medical Subject Headings (MeSH): "Mental Disorders", "Family", "Caregivers". A operacionalização e a estratégia de busca se deram a partir da combinação com operador booleano AND
Quadro 1 - Estratégia de busca por base de dados. Recife, Pernambuco (PE), Brasil, 2021.

\begin{tabular}{clcc}
$\begin{array}{c}\text { Bases de } \\
\text { dados }\end{array}$ & \multicolumn{1}{c}{ Termos da busca } & Resultados & $\begin{array}{c}\text { Seleciona- } \\
\text { dos }\end{array}$ \\
\hline DOAJ & Mental Disorders AND Family AND Caregivers & 160 & 3 \\
\hline LILACS & Mental Disorders AND Family AND Caregivers & 123 & 1 \\
\hline MEDLINE & Mental Disorders AND Family AND Caregivers & 3056 & 0 \\
\hline SciELO & Mental Disorders AND Family AND Caregivers & 28 & 1 \\
\hline SCOPUS & Mental Disorders AND Family AND Caregivers & 834 & 1 \\
\hline $\begin{array}{l}\text { Web of } \\
\text { Science }\end{array}$ & Mental Disorders AND Family AND Caregivers & 1539 & 3 \\
\hline Total & & 5740 & 9
\end{tabular}

Fonte: Dados da pesquisa, 2021.

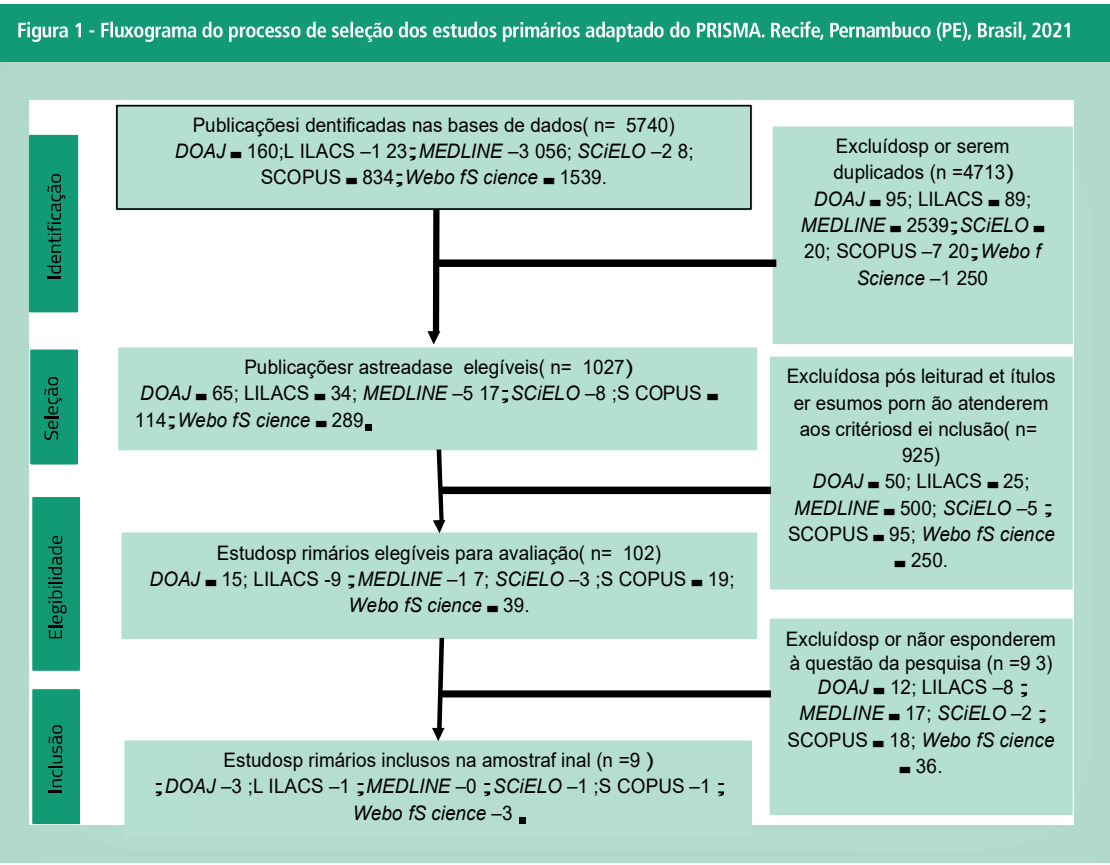

Fonte: Dados da pesquisa, 2021

e OR, efetuando a busca conjunta e individualmente para que possíveis diferenças fossem corrigidas (Quadro 1).

Os artigos foram selecionados baseando-se no Preferred Reporting Items for Systematic Review and Meta-Analyses (PRISMA) com o objetivo de auxiliar no desenvolvimento de artigos (7). A princípio eliminaram-se por meio da leitura de títulos e resumos, estudos duplicados. Destes pré-selecionados, realizou-se leitura na íntegra, a fim de verificar os que atendem à questão norteadora e aos critérios de inclusão/ exclusão. Construiu-se então a amostra final com estudos pertinentes aos critérios pré-estabelecidos (Figura 1).

Consolidada a leitura dos artigos, os selecionados foram categorizados, classificando o conhecimento produzido em níveis de evidência de acordo com Melnyk \& Fineout-Overholt (8): nível I, as evidências são relacionadas à revisão sistemática ou metanálise de ensaios clínicos randomizados controlados ou provenientes de diretrizes clínicas baseadas em revisões sistemáticas de ensaios clínicos randomizados 
controlados; no nível II, evidências derivadas de no mínimo um ensaio clínico randomizado controlado bem delineado; no nível III, evidências de ensaios clínicos bem delineados sem randomização; no nível IV, evidências advindas de estudos de coorte e de caso-controle bem delineados; no nível $\mathrm{V}$, evidências provenientes de revisão sistemática de estudos descritivos e qualitativos; no nível VI, evidências derivadas de um único estudo descritivo ou qualitativo; e no nível VII, evidências derivadas de opinião de autoridades e/ou relatório de comitês de especialistas.

Foi elaborada a sumarização das informações do corpus através de um instrumento: identificação do artigo original; autoria do artigo; ano de publicação; país; características metodológicas do estudo; e amostra do estudo. Permitindo-se obter conclusões gerais devidos à reunião de vários estudos, por meio do processo de análise sistemática e síntese da literatura pesquisada. Quando bem elaborada pode representar o estado atual da literatura pesquisada. Além disso, evidencia as lacunas de conhecimento que precisam ser preenchidas com a realização de novos estudos.

Para que houvesse uma melhor compreensão e visualização dos principais achados organizaram-se os dados apresentando-os em figuras e tabelas, expostos de forma descritiva.

\section{RESULTADOS}

Os estudos selecionados estão dispostos de modo a evidenciar seus títulos, autores, anos de publicação, níveis de evidências, objetivos e resultados. Após a leitura dos artigos selecionados, os estudos foram categorizados, classificando o conhecimento produzido sobre o tema, em níveis de evidência, em sua maioria nível VI - evidências derivadas de um único estudo descritivo ou qualitativo. Os principais achados dispostos nos objetivos e conclusões,

66

A família é
constituída com
base nas relações
de parentesco
cultural, sendo
historicamente
determinada,
ncluindo-se entre as

instituições básicas, apontada como elemento-chave não apenas para a sobrevivência dos indivíduos, mas também para a proteção e a socialização de seus componentes, transmissão do capital cultural e econômico e da propriedade de grupo $[\ldots]$ estão diretamente associados aos desafios enfrentados pela família cuidando da pessoa com transtorno mental ( $\mathrm{Ta}$ bela 1).

Dado o exposto, observa-se que dentre os maiores desafios enfrentados pelos cuidadores familiares está a sobrecarga, principalmente nos cuidadores do sexo feminino. Estes que se veem sozinhos e muitas vezes sem o apoio da equipe de saúde. Apoio este que se faz imprescindível desde o primeiro momento, visando a saúde não só do paciente como também de seu cuidador.

\section{DISCUSSÃO}

Ao término das buscas nas bases de dados, seleção dos artigos, leitura e análise dos mesmos, evidenciou-se a categorização de termos, os quais retratam e descrevem o que traz a literatura acerca dessa temática, mas principalmente as respostas e os enfrentamentos vivenciados pelos familiares das pessoas com transtorno mental, as dificuldades enfrentadas por eles, e sobretudo para essa pessoa que vive com transtorno mental o fato de como é difícil sua (re) inserção no seio familiar e na sociedade após longos anos fora do contexto social e familiar. A seguir estão descritas algumas categorias encontradas.

\section{Afastamento familiar}

A família é constituída com base nas relações de parentesco cultural, sendo historicamente determinada, incluindo-se entre as instituições básicas, apontada como elemento-chave não apenas para a sobrevivência dos indivíduos, mas também para a proteção e a socialização de seus componentes, transmissão do capital cultural e econômico e da propriedade de grupo, bem como das relações de gênero e de solidariedade entre gerações. Por meio dela, incorporam-se padrões de comportamento, valores morais, sociais, 


Tabela 1 - Síntese dos principais achados sobre os desafios enfrentados pela família cuidando da pessoa com transtorno
mental. Recife, Pernambuco (PE), 2021.

Challenges of Providing
Home Care for a Family
Member with Serious
Chronic Mental Illness: A
Qualitative Enquiry. / DOAJ

Mokwena,

Kebogile Elizabeth; Ngoveni, Suiça ॥ mento domiciliar a pacientes Amukelane. com transtornos mentais

(2020)
A ausência de habilidades e recursos necessários para cuidar de doentes mentais em casa expõe os pacientes e suas famílias a dificuldades emocionais, financeiras e sociais, e resulta em desfechos desfavoráveis tanto para os pacientes quanto para suas famílias.

\begin{tabular}{|c|c|c|c|c|c|c|}
\hline 3 & $\begin{array}{l}\text { An Exploration of Family } \\
\text { Caregiver Experiences of } \\
\text { Burden and Coping While } \\
\text { Caring for People with } \\
\text { Mental Disorders in Saudi } \\
\text { Arabia_A Qualitative } \\
\text { Study. / DOAJ }\end{array}$ & $\begin{array}{l}\text { Sharif, Loujain } \\
\text { et al. (2020) }\end{array}$ & $\begin{array}{l}\text { Arábia } \\
\text { Sau- } \\
\text { dita }\end{array}$ & $\mathrm{VI}$ & $\begin{array}{l}\text { Explorar as vivências de cui- } \\
\text { dadores familiares de pessoas } \\
\text { com transtorno mental, por } \\
\text { meio do exame das cargas } \\
\text { que enfrentam e das estraté- } \\
\text { gias de enfrentamento que } \\
\text { utilizam. }\end{array}$ & $\begin{array}{l}\text { Os cuidadores familiares de pessoas com } \\
\text { transtornos mentais enfrentam uma série de } \\
\text { fardos e fatores de estresse, como estigma } \\
\text { associativo e esgotamento. Muitas vezes, } \\
\text { essas cargas são decorrentes de seu papel } \\
\text { de cuidar aliado a suporte insuficiente ou } \\
\text { estratégias de enfrentamento ineficazes, } \\
\text { que podem afetar sua qualidade de vida e } \\
\text { integridade biopsicossocial que, por sua vez, } \\
\text { podem afetar o cuidado que prestam. }\end{array}$ \\
\hline 4 & $\begin{array}{l}\text { Sobrecarga de cuidadores } \\
\text { familiares de pessoas com } \\
\text { transtornos mentais: aná- } \\
\text { lise dos serviços de saúde. } \\
\text { / LILACS }\end{array}$ & $\begin{array}{l}\text { Eloia, Sara } \\
\text { Cordeiro et al. } \\
\quad(2018)\end{array}$ & Brasil & $\mathrm{VI}$ & $\begin{array}{l}\text { Analisar o grau de sobrecarga } \\
\text { objetiva e subjetiva sentida } \\
\text { por cuidadores familiares de } \\
\text { pacientes psiquiátricos. }\end{array}$ & $\begin{array}{l}\text { Apresentou-se maior sobrecarga com relação } \\
\text { à supervisão aos comportamentos problemá- } \\
\text { ticos e no impacto nas rotinas diárias. }\end{array}$ \\
\hline 5 & $\begin{array}{l}\text { Minor psychiatric disorders } \\
\text { and their associations in } \\
\text { family caregivers of people } \\
\text { with mental disorders. / } \\
\text { SciELO }\end{array}$ & $\begin{array}{l}\text { Treichel, } \\
\text { Carlos Alberto } \\
\text { dos Santos et } \\
\text { al. (2017) }\end{array}$ & Brasil & $\mathrm{VI}$ & $\begin{array}{c}\text { Analisar a ocorrência de trans- } \\
\text { tornos psiquiátricos menores e } \\
\text { suas associações em familiares } \\
\text { de pessoas com transtornos } \\
\text { mentais. }\end{array}$ & $\begin{array}{l}\text { Maior prevalência desses transtornos esteve } \\
\text { fortemente associada ao sexo feminino, } \\
\text { idade avançada, laços familiares de primeiro } \\
\text { grau, não possuir trabalho remunerado, } \\
\text { nivel de educação mais baixo, menor renda, } \\
\text { problemas de saúde, baixa qualidade de vida } \\
\text { e sentimento de sobrecarga. }\end{array}$ \\
\hline
\end{tabular}

Barriers to Family Caregi$6 \quad$ vers' Coping With Patients With Severe Mental Illness in Iran. / SCOPUS
Ebrahimi,

Hossein et al. Irã $\quad$ V|

(2018)
Identificar o amplo espectro de problemas causados por cuidar de um paciente com mental doença impõe uma grande carga sobre família cuidadores
Os resultados consistiram em quatro categorias principais: 0 isolamento do paciente da vida cotidiana, recuperação incompleta, falta de apoio por parte do sistema de saúde e estigmatização. 


\begin{tabular}{|c|c|c|c|c|c|c|}
\hline 7 & $\begin{array}{l}\text { The experience of caregi- } \\
\text { vers of people living with } \\
\text { serious mental disorders: a } \\
\text { study from rural Ghana. / } \\
\text { Web Of Science }\end{array}$ & $\begin{array}{l}\text { Ae-ngibise, } \\
\text { Kenneth } \\
\text { Ayuurebobi et } \\
\text { al. (2015) }\end{array}$ & Gana & $\mathrm{VI}$ & $\begin{array}{l}\text { Avaliar a carga de cuidados } \\
\text { sobre os cuidadores de pesso- } \\
\text { as que vivem com transtornos } \\
\text { mentais. }\end{array}$ & $\begin{array}{l}\text { Sofrimento emocional, estigma, sobrecarga } \\
\text { financeira, falta de redes de apoio, exclusão } \\
\text { social, impacto na saúde e ausência de servi- } \\
\text { ços de saúde mental descentralizados foram } \\
\text { vivenciados por cuidadores familiares. }\end{array}$ \\
\hline 8 & $\begin{array}{l}\text { Family caregiver burden in } \\
\text { mental illnesses: The case } \\
\text { of affective disorders and } \\
\text { schizophrenia - a qualitati- } \\
\text { ve exploratory study. / Web } \\
\text { Of Science }\end{array}$ & $\begin{array}{l}\text { Von Kardorff, } \\
\text { Ernst et al. } \\
(2015)\end{array}$ & $\begin{array}{c}\text { Alema- } \\
\text { nha }\end{array}$ & $\mathrm{VI}$ & $\begin{array}{l}\text { Explorar as cargas específicas } \\
\text { vivenciadas por cuidadores de } \\
\text { pacientes com esquizofrenia e } \\
\text { transtornos afetivos. }\end{array}$ & $\begin{array}{l}\text { Cuidar de uma pessoa com doença mental } \\
\text { afeta os cuidadores emocionalmente, finan- } \\
\text { ceiramente, fisicamente e provoca algumas } \\
\text { restrições em sua rotina (aborrecimentos } \\
\text { diários). Por fim, causa conflitos nas relações } \\
\text { familiares. }\end{array}$ \\
\hline 9 & $\begin{array}{l}\text { Factors associated with the } \\
\text { burden of family caregivers } \\
\text { of patients with mental } \\
\text { disorders: a cross-sectional } \\
\text { study. / Web of Science }\end{array}$ & $\begin{array}{l}\text { Souza, Ana } \\
\text { Lúcia Rezende } \\
\text { et al. (2017) }\end{array}$ & Brasil & $\mathrm{VI}$ & $\begin{array}{l}\text { Identificar fatores associados } \\
\text { à sobrecarga de cuidadores } \\
\text { de familiares com transtornos } \\
\text { mentais. }\end{array}$ & $\begin{array}{l}\text { Os fatores independentemente associados à } \\
\text { sobrecarga dos cuidadores foram depressão, } \\
\text { ter mais de } 60 \text { anos de idade, não receber } \\
\text { ajuda para cuidar, crise recente do paciente, } \\
\text { dias de contato e ter outros membros da } \\
\text { família necessitando de cuidados. }\end{array}$ \\
\hline
\end{tabular}

Fonte: Dados da pesquisa, 2021

éticos e espirituais (9).

A família da pessoa em sofrimento psíquico, a partir do surgimento da psiquiatria enquanto saber médico responsável pelo desvelamento da loucura, ficou afastada do tratamento de seu parente, visto que ele se encontrava em hospital psiquiátrico. Nesse modelo, o familiar era alijado do tratamento, sentindo-se culpado devido às proibições de visitas (10).

$\mathrm{Na}$ institucionalização da loucura, na qual o saber médico passa a ser o detentor e dominador do então "doente mental", o afastamento dos indivíduos do seu meio social e familiar tornou-se uma premissa do tratamento proposto naquela época, pois a família era vista como causadora do adoecimento, reforçando ainda mais a necessidade do isolamento enquanto medida terapêutica. A relação da família com o manicômio era de cumplicidade na internação do paciente, traduzida na gratidão desses familiares em verem-se aliviados do problema (11).

Com a Reforma psiquiátrica e a desinstitucionalização dos hospitais psiquiátricos, o cuidador passa a ser a família ou o parente que assume esse papel, sendo estes responsáveis pela pessoa com transtorno mental. Entende-se por cuidador familiar a pessoa que, por vínculos parentais, assume a responsabilidade, direta ou não, pelo cuidado de um familiar doente e/ou dependente (12).

\section{Conflitos no contexto familiar}

Os conflitos familiares são eminentes quando a família vivencia situação de enfermidade de um familiar, pois as diferenças quanto à disponibilidade para o cuidado, bem como ao interesse e aptidão para o mesmo, podem levar à necessidade de intervenção por parte de um profissional de saúde por meio de uma postura clínica e de orientações constantes para o cuidado do paciente, a qual pode amenizar a vivência do cuidado realizado pelo cuidador, que contribui para a diminuição dos conflitos familiares em relação ao doente e suas necessidades de cuidado (13).

Ressalta-se que a sobrecarga dos cuidadores familiares é um fator estressante. O cuidador assume múltiplas funções tornando-se o "cuidador único", eventualmente auxiliado em tarefas menores por outros membros da família, fato que o leva ao desgaste físico e mental, podendo prejudicar o controle da doença e a qualidade de vida não apenas do doente, mas de toda a família (14).

Nesta situação, os conflitos de toda ordem podem ser amenizados quando o profissional de saúde cuida, mas também identifica o cuidador principal, orientando as necessidades de cuidados direto e indireto a essa pessoa, contribuindo com a diminuição da ansiedade e angústia vivenciada pela família como um todo. Uma pessoa que cuida de outra pessoa no decorrer do processo vivenciado vai se despersonificando, vai perdendo as características familiares e vai progressivamente assumindo outro modo de ser (14).

\section{Saúde mental e cidadania}

A Lei Federal $n^{\circ} 10.216$, de 06 de dezembro de 2001, garante os direitos das pessoas com transtorno mental, incluindo aqueles relacionados ao uso de substâncias psicoativas. De forma geral, a Lei $\mathrm{n}^{\circ}$ 10.216/2001 assegura às pessoas que se beneficiam das ações e serviços do SUS o direito a um tratamento que respeite a sua cidadania e que por isso deve ser realizado de preferência em serviços comunitários, ou de base territorial, portanto, sem excluí-las do convívio na sociedade (14, 15).

É primordial, então, incluir ativamente nas políticas de expansão, formulação e avaliação da Atenção Primária à Saúde as Ações de Saúde Mental 
que, com potencial transversal, devem ajudar as equipes a trabalhar a dimensão do sofrimento psíquico, o qual é mais que uma simples ameaça à integridade biológica, como também à sua integridade como ser humano, como sujeito de ação e reação, que possui necessidades próprias e específicas (15-17).

Realizando as estratégias de busca encontrou-se uma amostra pequena e pouca disponibilidade de artigos científicos para comparar os resultados. Bem como foram levantados poucos artigos como resultado dos descritores, poucos atenderam ao objetivo do estu- do. Ainda, os estudos incluídos apresentam limitações como: unicêntricos, diferentes sistemas de comparação, tamanho pequeno da amostra e falta de randomização.

Assim, não se pode comprovar a existência de evidência científica relacionada aos desafios enfrentados pela família cuidando da pessoa com transtorno mental. Fazendo-se necessário a realização de mais estudos contendo uma amostra maior e possibilitando discussão acerca dos aspectos associados ao tema.

CONCLUSÃO
Observou-se nesta revisão os desafios enfrentados pela família cuidando da pessoa com transtorno mental; associados principalmente, ao despreparo dos profissionais e dos familiares com a reforma psiquiátrica e a desinstitucionalização. No entanto, mesmo que ainda haja escassez de estudos que dão a verdadeira importância a este tema, percebe-se quão grande é o déficit de conhecimento dos profissionais a respeito do que estava por vir e dos familiares que não conheciam a doença e não estavam preparados para dar o suporte necessário.

\section{Referências}

1. Sampaio ML, Bispo JP. Entre o enclausuramento e a desinstitucionalização: a trajetória da saúde mental no Brasil. Trabalho, Educação e Saúde. 2021, 19: e00313145. https://doi.org/10.1590/1981-7746-sol00313.

2. Delgado P. Reforma psiquiátrica: estratégias para resistir ao desmonte. Trabalho, Educação e Saúde, Rio de Janeiro, 2019; 17(2): e0021241. https://doi.org/10.1590/1981-7746-sol00212

3. Clementino FS, Miranda FAN, Pessoa Júnior JM, Marcolino EC, Silva Júnior JÁ, Brandão GCG. Atendimento integral e comunitário em saúde mental: Avanços e desafios da Reforma Psiquiátrica. Trabalho, Educação e Saúde, Rio de Janeiro, 2019; 17(1) :e0017713. https://doi.org/10.1590/ 1981-7746-sol00177

4. Fernandes CJ. Reforma psiquiátrica (im)possível? estudo documental e analítico (2008 a 2017). 2018. 137f. Dissertação (Mestrado em Psicologia) - Programa de Pós-graduação em Psicologia, Universidade Federal do Ceará, Ceará, 2018.

5. Ercole FF, Melo LS, Alcoforado CLGC. Integrative review versus systematic review. Reme: Revista Mineira de Enfermagem, 2014; 18(1), 2014. GN1 Genesis Network. http://doi.org/10.5935/1415-2762.20140001

6. Lisboa MT. Elementos para elaboração de um desenho de pesquisa | Elements to formulate a research design. Mural Internacional, 2019; 10:38439-1. Universidade de Estado do Rio de Janeiro. http://doi. org/10.12957/rmi.2019.38439

7. Barbosa FT, Lira AB, Oliveira Neto OB, Santos LL, Santos IO, Barbosa LT et al. Tutorial para realizar revisão sistemática e meta-análise com estudos de anestesia intervencionista. Rev. Bras. Anestesiol. Junho de 2019;69(3): 299-306. https://doi.org/10.1016/j.bjane.2019.03.003

8. Melnyk BM.; Fineout-Overholt E. Making the case for evidence-based practice. In B. M. MELNYK \& E. FINEOUT-OVERHOLT. Evidence-based practice in nursing \& healthcare: a guide to best practice. Philadelphia: Lippincot Williams \& Wilkins. 2005; 3-24.

9. Lima MC, Goncalves TR. Apoio matricial como estratégia de ordenação do cuidado em saúde mental. Trabalho, Educação e Saúde, Rio de Janeiro, 2020; 18(1): e0023266. https://doi.org/10.1590/1981-7746-sol00232

10. Machado CV, Lima LD, Baptista TWDF. Políticas de saúde no Brasil em tempos contraditórios: caminhos e tropeços na construção de um sistema universal. Cadernos de Saúde Pública, Rio de Janeiro, 2017; 33: e00129616, Suplemento 2. https://doi.org/10.1590/0102-311X00129616 11. Mezzina R. Forty years of the Law 180: the aspirations of a great reform, its successes and continuing need. Epidemiology and psychiatric sciences, Cambrigde, 2018; 27(4): 336-345. https://doi.org/10.1017/ S2045796018000070

12. Vieira FS, Santos IS, Ocké-Reis CO, Rodrigues PHA. Políticas Sociais e Austeridade Fiscal: como as Políticas sociais são afetadas pelo austericídio da agenda neoliberal no Brasil e no mundo Rio de Janeiro: Cebes; 2018.

13. Rodrigues PHA, Santos IS. Os novos riscos sociais não são só europeus, também chegaram ao Brasil e exigem respostas das nossas políticas sociais. In: Rodrigues PHA, Santos IS, organizadores. Políticas e riscos sociais no Brasil e na Europa: convergências e divergências Rio de Janeiro: Cebes; Editora Hucitec; 2017. p. 111-141.

14. Giovanella LG, Mendoza-Ruiz A, Pilar ACA, Rosa MC, Martins GB, Santos IS, Barata D, Vieira JMDL, Castro VG, Silva PO, Rohem ACR, Machado CV. Sistema universal de saúde e cobertura universal: desvendando pressupostos e estratégias. Cien Saude Colet 2018; 23(6):1763-1776. https:/l doi.org/10.1590/1413-81232018237.09192018

15. Onocko-Campos RT, Amaral CEM, Saraceno B, de Oliveira BDC, Treichel CADS, Delgado PGG. Atuação dos Centros de Atenção Psicossocial em quatro centros urbanos no Brasil. Rev Panam Salud Publica. 2018; 42:e113. https://doi.org/10.26633/RPSP.2018.113

16. Santos IS, Vieira FS. Direito à saúde e austeridade fiscal: o caso brasileiro em perspectiva internacional. Ciência \& Saúde Coletiva, Rio de Janeiro, 2018; 23(7): 2.303-2.314. https://doi.org/10.1590/141381232018237.09192018

17. Piedade FL, Santos PA dos, Haddad JGV. Os significados acerca da Estratégia Saúde da Família para uma comunidade. Nursing. $1^{\circ}$ de fevereiro de 2021; 24(273):5219-28. https://doi.org/10.36489/nursing. 2021v24i273p5219-5228 